\title{
Caractérisation d'un ensemble généralisant l'ensemble des nombres de Pisot
}

\author{
par
}

\author{
TOUfIK ZAÏMI (Riyadh)
}

1. Introduction. Soient $K$ un corps de nombres et $\theta$ un entier algébrique de module $>1$ et de polynôme minimal $\operatorname{Irr}(\theta, K, z)$ sur $K$. Alors $\theta$ est dit $K$-nombre de Pisot si pour tout plongement $\sigma$ de $K$ dans $\mathbb{C}$ le polynôme $\sigma \operatorname{Irr}(\theta, K, z)$ possède une unique racine de module $>1$ et aucune racine de module 1. Ces nombres ont été définis par A. M. Bergé et J. Martinet [2]. Comme dans [2], on représente un $K$-nombre de Pisot $\theta$ dans l'algèbre $A=\mathbb{R}^{r_{1}} \times \mathbb{C}^{r_{2}}$, où $\left(r_{1}, r_{2}\right)$ désigne la signature du corps $K$, par la suite $\left(\theta_{\sigma}\right)_{\sigma}$ de ses conjugués de module $>1$ et on note $S_{K}$ leur ensemble dans $A$. D'après le théorème 1 de [7], l'ensemble $S_{K}$ est fermé dans $A$ seulement lorsque $K=\mathbb{Q}$ ou bien $K=\mathbb{Q}(\sqrt{d})$ où $d \in \mathbb{Z}^{-}$. On peut espérer obtenir dans $A$ un ensemble fermé d'entiers algébriques généralisant l'ensemble $S_{\mathbb{Q}}$ en rajoutant aux éléments de $S_{K}$ les points limites suivant la preuve du théorème 1 de [7] et l'on obtient alors un ensemble $\Sigma_{K}$ qu'on peut définir comme étant l'ensemble des entiers algébriques $\theta$ de module $>1$ tels que pour tout plongement $\sigma$ le polynôme $\sigma \operatorname{Irr}(\theta, K, z)$ admet au plus une racine de module $>1$ et aucune racine de module 1 . L'ensemble $\Sigma_{K}$ coïncide avec l'ensemble $S_{K}$ seulement lorsque $K=\mathbb{Q}$ ou bien $K=\mathbb{Q}(\sqrt{d})$ où $d<0$ et dans ces cas il est fermé. On donne ici une caractérisation de cet ensemble.

2. Les résultats. La caractérisation suivante de l'ensemble $\Sigma_{\mathbb{Q}}$ est dûe à C. Pisot :

ThÉORÈme A [4]. Soit $\theta$ un nombre complexe de module $>1$. Alors $\theta \in \Sigma_{\mathbb{Q}}$ si et seulement si il existe un nombre complexe non nul $\lambda$ et une suite $\left(a_{n}\right)_{n}$ d'entiers de $\mathbb{Q}$ tels que

$$
\sum_{n \geq 0}\left(\lambda \theta^{n}-a_{n}\right)^{2}<\infty
$$

1991 Mathematics Subject Classification: Primary 11R06. 
Une autre caractérisation de l'ensemble $\Sigma_{\mathbb{Q}}$ démontrée par C. Pisot et T. Vijayaraghavan est la suivante :

ThÉORÈme B [5]. Soit $\theta$ un nombre complexe algébrique de module $>1$. Alors $\theta \in \Sigma_{\mathbb{Q}}$ si et seulement si il existe un nombre complexe non nul $\lambda$ et une suite $\left(a_{n}\right)_{n}$ d'entiers de $\mathbb{Q}$ tels que

$$
\lim _{n}\left(\lambda \theta^{n}-a_{n}\right)=0 .
$$

Avec les notations précédentes et si on représente dans $A$ un élément $\theta$ de $\Sigma_{K}$ par la suite $\left(\theta_{\sigma}\right)_{\sigma}$ où $\theta_{\sigma}$ est soit la racine de module $>1$ du polynôme $\sigma \operatorname{Irr}(\theta, K, z)$ lorsqu'elle existe, soit 0 , on obtient alors le résultat suivant :

ThÉORÈme 1. Soit $\theta \in \Sigma_{K}$; il existe alors un nombre complexe non nul $\lambda$ dans $K(\theta)$ et une suite $\left(a_{n}\right)_{n}$ d'entiers de $K$ tels que

$$
\sum_{\sigma} \sum_{n \geq 0}\left|\sigma \lambda \theta_{\sigma}^{n}-\sigma a_{n}\right|^{2}<\infty .
$$

Ce théorème a été prouvé pour l'ensemble $S_{K}$ lorsque le corps $K$ est totalement réel dans [3] et la preuve donnée allait dans le sens de l'application de l'algorithme de Schur. On donne ici une preuve simple de ce résultat. De la dernière inégalité on déduit $\lim _{n}\left|\sigma \lambda \theta_{\sigma}^{n}-\sigma a_{n}\right|=0$ pour tout $\sigma$ et par suite le théorème 1 montre une des implications des théorèmes $\mathrm{A}$ et $\mathrm{B}$.

Réciproquement, si $d$ désigne le degré du corps $K$ sur $\mathbb{Q}$ on a alors :

THÉORÈme 2. (i) Soient d nombres complexes $\left(\theta_{\sigma}\right)_{\sigma}$ tels que $\left|\theta_{\sigma}\right|>1$ ou bien $\theta_{\sigma}=0$. On suppose l'existence d'une suite $\left(a_{n}\right)_{n}$ d'entiers de $K$ et de $d$ nombres complexes $\left(\lambda_{\sigma}\right)_{\sigma}$ tels que $\prod_{\sigma} \lambda_{\sigma} \neq 0$ et $\sum_{\sigma \in G} \sum_{n \geq 0}\left|\lambda_{\sigma} \theta_{\sigma}^{n}-\sigma a_{n}\right|^{2}$ $<\infty$. Alors $\theta_{\sigma} \in \Sigma_{\sigma K}$ pour tout plongement $\sigma$ tel que $\left|\theta_{\sigma}\right|>1$.

(ii) Soient $d$ nombres complexes algébriques $\left(\theta_{\sigma}\right)_{\sigma}$ tels que $\left|\theta_{\sigma}\right|>1$ ou bien $\theta_{\sigma}=0$. On suppose l'existence d'une suite $\left(a_{n}\right)_{n}$ d'entiers de $K$ et de $d$ nombres complexes $\left(\lambda_{\sigma}\right)_{\sigma}$ tels que $\prod_{\sigma} \lambda_{\sigma} \neq 0$ et $\lim _{n}\left(\lambda_{\sigma} \theta_{\sigma}^{n}-\sigma a_{n}\right)=0$ pour tout $\sigma$. Alors $\theta_{\sigma} \in \Sigma_{\sigma K}$ pour tout plongement $\sigma$ tel que $\left|\theta_{\sigma}\right|>1$.

Le théorème 2(i) a été également prouvé pour l'ensemble $S_{K}$ lorsque le corps $K$ est totalement réel dans [3].

\section{Preuve des théorèmes}

Preuve du théorème 1. Avec les notations précédentes, soient $\operatorname{Irr}(\theta, K, z)$ $=z^{s}-u_{1} z^{s-1}+u_{2} z^{s-2}+\ldots+(-1)^{s} u_{s}$ et $\alpha_{1, \sigma}, \alpha_{2, \sigma}, \ldots, \alpha_{s, \sigma}$ les racines du polynôme $\sigma \operatorname{Irr}(\theta, K, z)$. Par induction sur les identités de Newton : $a_{k, \sigma}=$ $\left(\sigma u_{1}\right) a_{k-1, \sigma}-\left(\sigma u_{2}\right) a_{k-2, \sigma}+\ldots+(-1)^{k+1} k \sigma u_{k}$, où $a_{k, \sigma}=\alpha_{1, \sigma}^{k}+\alpha_{2, \sigma}^{k}+$ $\ldots+\alpha_{s, \sigma}^{k}$ et $k \geq 1$, on obtient alors $a_{k, \sigma}=\sigma a_{k}$ où $a_{k}=a_{k, I}$ est un entier de $K, I$ désignant l'identité de $\operatorname{Gal}(K / \mathbb{Q})$. 
La série $\sum_{n>0}\left|\sigma \lambda \theta_{\sigma}^{n}-\sigma a_{n}\right|^{2}$ où $\theta_{\sigma}$ désigne ou bien la racine de module $>1 \mathrm{du}$ polynôme $\sigma \operatorname{Irr}(\theta, K, z)$ lorsqu'elle existe ou bien 0 et où $\lambda=1$, converge alors comme une série géométrique pour tout $\sigma$; d'où le résultat.

Preuve du théorème 2. (i) Considérons le déterminant

$$
\Delta_{n, \sigma}=\left|\begin{array}{cccc}
\sigma a_{0} & \sigma a_{1} & \ldots & \sigma a_{n} \\
\sigma a_{1} & \sigma a_{2} & \ldots & \sigma a_{n+1} \\
\vdots & & & \\
\sigma a_{n} & \sigma a_{n+1} & \ldots & \sigma a_{2 n}
\end{array}\right| .
$$

Le développement de $\Delta_{n, \sigma}$ montre que les $\left(\Delta_{n, \sigma}\right)_{\sigma}$ sont des entiers algébriques conjugués sur $\mathbb{Q}$. De manière identique à la preuve dans [4], on obtient la majoration $\left|\Delta_{n, \sigma}\right|<1$ à partir d'un certain rang $N$ ne dépendant pas de $\sigma$ et par suite les $\left(\Delta_{n, \sigma}\right)_{\sigma}$ sont tous nuls à partir d'un certain rang. Le critère de rationalité de Kronecker montre alors que la série $\sum_{n \geq 0} \sigma a_{n} z^{n}$ est récurrente pour tout plongement $\sigma$. Le lemme de Fatou généralisé [1] montre qu'on peut écrire la série $\sum_{n>0} \sigma a_{n} z^{n}$ sous la forme $\sum \sigma a_{n} z^{n}=$ $R_{\sigma}(z) / Q_{\sigma}(z)$ où $R_{\sigma}$ et $Q_{\sigma}$ sont deux polynômes à coefficients entiers de $\sigma K$, premiers entre eux et tels que $Q_{\sigma}(0)=1$. Soit $f_{\sigma}$ la fonction définie par

$$
f_{\sigma}(z)=\frac{\lambda_{\sigma}}{1-\theta_{\sigma} z}-\sum_{n \geq 0} \sigma a_{n} z^{n}=\sum_{n \geq 0}\left(\lambda_{\sigma} \theta_{\sigma}^{n}-\sigma a_{n}\right) z^{n} \quad \text { lorsque }\left|\theta_{\sigma}\right|>1 .
$$

Comme cette fonction a un rayon de convergence au moins égal à 1 , le polynôme $Q_{\sigma}$ admet une seule racine $1 / \theta_{\sigma}$ de module $<1$ et comme $f_{\sigma}$ est sans pôle de module 1 , les autres racines de $Q_{\sigma}$ sont de module $>1$. Soit $P_{\sigma}$ le polynôme unitaire à coefficients entiers de $\sigma K$ défini par $P_{\sigma}(z)=$ $z^{q} Q_{\sigma}(1 / z)$ où $q$ est le degré de $Q_{\sigma}$. Si $\theta_{\sigma}=0$ alors les racines de $P_{\sigma}$ sont toutes de module $<1$ et si $\left|\theta_{\sigma}\right|>1$ alors $\theta_{\sigma}$ est la seule racine de module $>1$ du polynôme $P_{\sigma}$, ses autres racines sont de module $<1$. Si on note $R(z) / Q(z)=\sum_{n \geq 0} a_{n} z^{n}$ alors

$$
\frac{\sigma R(z)}{\sigma Q(z)}=\sum_{n \geq 0} \sigma a_{n} z^{n}=\frac{R_{\sigma}(z)}{Q_{\sigma}(z)} .
$$

Comme les fractions $\sigma R / \sigma Q$ et $R_{\sigma} / Q_{\sigma}$ sont irréductibles on déduit que $Q_{\sigma}$ divise $\sigma Q$ et $\sigma Q$ divise $Q_{\sigma}$. De l'égalité $Q_{\sigma}(0)=\sigma Q(0)=1$ on déduit $Q_{\sigma}(z)=\sigma Q(z)$, d'où le résultat.

(ii) Soit $\operatorname{Irr}\left(\theta_{\sigma}, \sigma K, z\right)=\sigma u_{0} z^{k}+\sigma u_{1} z^{k-1}+\ldots+\sigma u_{k}$ le polynôme minimal de $\theta_{\sigma}$ sur $\sigma K$, choisi tel que les $\left(u_{i}\right)_{0 \leq i \leq k}$ soient des entiers de $K$. Le polynôme $P$ défini par $P(z)=\prod_{\sigma} \sigma^{-1} \operatorname{Irr}\left(\theta_{\sigma}, \sigma K, z\right)$ où

$$
\sigma^{-1} \operatorname{Irr}\left(\theta_{\sigma}, \sigma K, z\right)=u_{0} z^{k}+u_{1} z^{k-1}+\ldots+u_{k},
$$

est alors à coefficients entiers de $K$. De l'égalité $\sigma q_{0}+\sigma q_{1} \theta_{\sigma}+\sigma q_{2} \theta_{\sigma}^{2}+\ldots+$ $\sigma q_{s} \theta_{\sigma}^{s}=0$ où les $\left(q_{i}\right)_{0 \leq i \leq s}$ sont tels que $P(z)=q_{0}+q_{1} z+\ldots+q_{s} z^{s}$, on 
déduit $\lambda_{\sigma} \sum \sigma q_{i} \theta_{\sigma}^{i+n}=0$. Si $\varepsilon_{\sigma, j}=\lambda_{\sigma} \theta_{\sigma}^{j}-\sigma a_{j}$ où $(j, n) \in \mathbb{N}^{2}$ alors

$$
\sum_{i=0}^{s} \sigma q_{i} \sigma a_{i+n}=-\sum_{i=0}^{s} \sigma q_{i} \varepsilon_{\sigma, i+n}
$$

De l'hypothèse on déduit alors l'existence d'un rang $N$ à partir duquel les entiers algébriques conjugués $\left(\sigma \sum_{i=0}^{s} q_{i} a_{i+n}\right)_{\sigma}$ sont tous de module $<1$ et donc nuls. La série $\sum_{n \geq 0} \sigma a_{n} z^{n}$ est alors récurrente pour tout plongement $\sigma$ et la suite de la preuve est identique à celle du théorème 2(i).

L'hypothèse $\lim _{n}\left(\lambda_{\sigma} \theta_{\sigma}^{n}-\sigma a_{n}\right)=0$ suffit pour que la fonction $f_{\sigma}$ définie dans la preuve du théorème 2(i) soit sans pôle de module 1 .

Remarque. Dans l'espoir d'obtenir des ensembles fermés d'entiers algébriques généralisant l'ensemble $S_{\mathbb{Q}}$, on peut enlever les points limites de l'ensemble $S_{K}$ suivant la preuve du théorème 1 de [7] en rajoutant une hypothèse du type $\theta \in S_{K}$ et $K \subset \mathbb{Q}(\theta)$. Toutefois d'après [6], si $K$ est un corps quadratique contenant une racine de l'unité non réelle $j$ alors la suite $\left(\theta_{n}\right)_{n}$ définie par $\left|\theta_{n}\right|>1$ avec $\theta_{n}$ racine du polynôme $z^{n}\left(z^{2}-z-1\right)+j\left(z^{2}-1\right)$ est une suite de $K$-nombres de Pisot qui vérifie $K \subset \mathbb{Q}\left(\theta_{n}\right)$ et converge vers le $K$-nombre de Pisot $\theta=(1+\sqrt{5}) / 2$ qui lui ne vérifie pas l'hypothèse $K \subset \mathbb{Q}(\theta)$.

\section{Bibliographie}

[1] B. Benzaghou, Anneaux de Fatou, Séminaire Delange-Pisot-Poitou, Théorie des nombres, 9-ième année (1968/69), no. 9, 8 p.

[2] A. M. Bergé et J. Martinet, Notions relatives de régulateurs et de hauteurs, Acta Arith. 54 (1989), 155-170.

[3] M. J. Bertin, K-nombres de Pisot et de Salem, ibid. 68 (1994), 113-131.

[4] C. Pisot, La répartition modulo 1 et les nombres algébriques, Ann. Scuola Norm. Sup. Pisa 7 (1938), 205-248.

[5] T. Vijayaraghavan, On the fractional parts of the powers of a number (II), Proc. Cambridge Philos. Soc. 37 (1941), 349-357.

[6] T. Zaïmi, Sur les nombres de Pisot relatifs, thèse de l'université Paris 6, Mai 1994.

[7] —, Sur la fermeture de l'ensemble des K-nombres de Pisot, Acta Arith. 83 (1998), $363-367$

Department of Mathematics

King Saud University

P.O. Box 2455

Riyadh 11451, Saudi Arabia

E-mail: zaimitou@ksu.edu.sa 